\title{
I DIRITTI FONDAMENTALI E LA LORO EFFETTIVITÀ
}

Francesco Bilancia ${ }^{1}$

\section{RIASSUNTO}

In questo saggio io intendo APPROCHARE il dibatitto sul tema dei diritti fondamentali, coinvolgendo la prospettiva di sua effettività principalmente nel sistema costituzionale europeo. Per tanto, si parte della approfundatta diferenzza che ci sono fra i membri statali e le multipli aspetti culturali e sociale della cittadinanzza europea.

Parole chiavi: Costituzione. Diritti Fondamentali. Società Europea.

1. Premessa. Un riflessione sul tema della effettività dei diritti nel sistema costituzionale europe ${ }^{2}$ comporta la necessità di misurarsi con una duplice difficoltà, impegnando a confrontarsi da un lato con una letteratura a dir poco sterminata oltre che articolata secondo approcci di analisi e metodi ricostruttivi estremamente differenziati; e comportando dall'altro il problema di muoversi nell'ambito di un sistema fenomenologico in rapida, costante evoluzione, versando le istituzioni comunitarie, e la stessa giurisprudenza della Corte di Giustizia in un contesto di rilevante transizione, per non considerare le più attuali vicende politiche connesse alla mancata ratifica del Trattato che istituisce una Costituzione per l'Europa ${ }^{3}$.

Delimito, quindi, convenzionalmente il tema a me affidato ad un'indagine circa il tenore delle disposizioni relative ai diritti fondamentali oggi raccolte nel Trattato costituzionale europeo ${ }^{4}$, frutto della incorporazione nel Trattato della Carta di Nizza del 2001, a sua volta sintesi degli standard di garanzia dei medesimi diritti secondo le contemporanee linee di sviluppo della giurisprudenza della Corte di giustizia. Le attuali difficoltà incontrate dalla giustizia europea nel sistematizzare in termini di effettività un catalogo certo di facoltà e limiti sintesi della concreta disciplina dei singoli diritti nel contesto europeo ${ }^{5}$ hanno, come noto, nelle riflessioni della dottrina immediato riscontro nel ricco ed elaborato quadro di soluzioni metodiche non sempre, invero, reciprocamente coerenti. II dato emerge dal complicato intreccio di 
ipotesi di ricostruzione scientifica dell'impianto di tali diritti oggi a disposizione degli studiosi, confrontandosi tra loro letture testuali delle disposizioni inscritte nella Carta di Nizza con più articolate ricostruzioni del processo di definizione di un vero e proprio sistema europeo dei diritti fondamentali; analisi aperte all'assimilazione anche dei dati politico-istituzionali deducibili dal contesto materiale di evoluzione e sviluppo delle fattispecie normative e giurisprudenziali ricognitive di tali diritti con approcci di carattere multilevel ${ }^{6}$, come dire condizionate dalla necessaria influenza che la concorrenza tra sistemi costituzionali esercita di fatto sulla struttura delle regole e sulla stessa resa dei sistemi di garanzia costituzionale dei diritti nel continuo confronto tra i diversi livelli di governo composti secondo lo schema teorico della rete.

Quest'ultimo approccio di metodo, che consente di riflettere sul ruolo della Corte di Giustizia, della Corte europea dei diritti dell'uomo (CEDU) e delle corti costituzionali nazionali come elementi di un sistema integrato deve quindi, di necessità, essere assunto come elemento di correzione dei distinti percorsi teorici che pretendano, da un lato, di guardare al Trattato costituzionale europeo come testo normativo a sé, isolato dal contesto politico-istituzionale di riferimento e passibile invece di un'interpretazione sistematico-formale come se si trattasse di una costituzione statale calata su un proprio conchiuso sistema politico di governo; e che indulgano, dall'altro, a costruire un polemico confronto tra trattato europeo e costituzioni nazionali al solo fine di isolare le differenze formali, anche qui senza tentare di ricostruire il contesto storico-politico teatro di questa evoluzione teorica ${ }^{7}$.

La compresenza di distinti percorsi teorici nella riflessione scientifica contemporanea è del resto sostenuta dal dato materiale stesso, laddove solo si osservi come a volte fattispecie ricognitive di nuovi diritti fondamentali dell'Unione europea si aggiungano al tradizionale catalogo contenuto nelle costituzionali nazionali; a volte procedano invece integrandosi nei sistemi nazionali con una funzione, per così dire, complementare; ed a volte si sostituiscano, invece, alle fattispecie ricognitive dei diritti costituzionali condizionandone la stessa efficacia in termini di funzionalizzazione degli stessi ad interessi superiori, comunque diversi rispetto a quelli propri dei titolari dei diritti ${ }^{8}$. Come esempio della prima tendenza segnalata, così, non può certo sfuggire all'interprete quale ampio spettro di novità 
siano oggi testualmente previste dalla Carta di Nizza, dal divieto di pena di morte e della tortura, ai nuovi diritti nel campo della medicina e della biotecnologia, dalle disposizioni a protezione della vita privata e familiare a quelle a protezione dei dati personali, fino a rinvenire norme a tutela del diritto di contrarre matrimonio e della proprietà intellettuale ${ }^{9}$. Come esempio della seconda tendenza, invece, ci si può forse limitare a segnalare la recente sentenza sul caso K.B. c. National Health Service Pensions Agency ${ }^{10}$ che ha condannato il Regno Unito a causa della proibizione legislativa per un transessuale di ottenere il mutamento degli atti dello stato civile, così incidendo negativamente sulla sua capacità di contrarre matrimonio, e ciò in violazione tra l'altro dell'art. 12 della Convenzione europea dei diritti dell'uomo, impedendo di fatto al coniuge convivente di ottenere il riconoscimento del diritto alla pensione di reversibilità. Dal divieto legale di contrarre matrimonio quale effetto dell'immodificabilità delle risultanze dell'atto di nascita la Corte ha, così, dedotto il venir meno della condizione per la concessione di un diritto retributivo aggiornando il catalogo dei divieti di discriminazione in base al sesso rispetto alla legislazione di uno Stato membro, ritenuta troppo restrittiva rispetto agli standards europei. Ed infine, la terza linea evolutiva della giurisprudenza europea in materia di diritti fondamentali, vale a dire l'ottica funzionalista quale matrice interpretativa del loro contenuto, è quanto mai evidente nella recente decisione sul caso Scmidberger c. Austria ${ }^{11}$, laddove la Corte ha proceduto ad un bilanciamento tra il diritto alla libertà di riunione in luogo pubblico e la libertà di circolazione delle merci (nel senso di scambi commerciali intracomunitari), operando una valutazione per equivalenti tra un diritto fondamentale della persona ed un elemento di struttura del mercato interno, non a caso qualificato dall'ordinamento europeo come libertà di circolazione. Tracce formali di funzionalizzazione al mercato dei diritti fondamentali sono, del resto, presenti nel testo stesso della Carta dei diritti come da subito segnalato da parte della dottrina ${ }^{12}$.

La compresenza di più linee di sviluppo dell'analisi, tra l'altro, suggerisce l'assunzione di forti cautele nel ricostruire teoriche dei diritti fondamentali da singoli casi giurisprudenziali se non al costo di inopportune semplificazioni sostenute da grave asistematicità, oltre che prodotto di esaltazione di obiter dicta astratti dal contesto delle problematiche di carattere materiale che qualificano la giurisprudenza comunitaria nella sua propria sostanza. 
E già in questa ottica l'analisi procederà mediante semplificazioni, non potendosi in questa sede affrontare il pur complesso intreccio tra la giurisprudenza creativa della Corte di Giustizia ed il catalogo dei diritti contenuto nella Carta di Nizza, che di quella giurisprudenza rappresenta in parte il precipitato attuale, in parte la proiezione futura ${ }^{13}$. L'arricchimento formale del catalogo dei diritti non consente, comunque, di mascherare il dato di una rilevata "cautela...sul versante della «effettività» dei diritti e dell'eguaglianza"14 usata nel recepimento da parte della Carta dei diritti della stessa giurisprudenza della Corte di Giustizia, implicando quindi di necessità l'onere di un'attenta osservazione dei contenuti della giurisprudenza, anche per evitare l'opposto rischio di una sua sistematizzazione forzosa. Sul fronte della effettività, infine, molto potrebbe offrire l'analisi delle garanzie che l'ordinamento europeo sia concretamente in grado di assicurare nei confronti dei diritti sociali, ma l'analisi della giurisprudenza della Corte di Giustizia in questo settore esula per ragioni di divisione dei compiti dalla mia riflessione. Per quanto connesso alla tutela dei diritti sociali rinvio, quindi, senz'altro alla Relazione del Prof. Gonzalo Maestro Buelga $^{15}$.

2. Diritti fondamentali e partecipazione politica. Un primo approccio di metodo nello studio dei diritti fondamentali guarda, come noto, alla struttura effettiva dell'ordinamento da cui questi siano riconosciuti e garantiti allo scopo di dedurre il grado di condizionamento che i principi dello «stato di diritto» assicurino agli strumenti di tutela dall'ordinamento stesso normativamente declinati. E' l'approccio che fa riferimento, insomma, alla fenomenologia della democrazia sostanziale quale riflesso del concreto operare degli istituti della democrazia procedurale, o formale nel quadro di una teoria normativa della Costituzione europea che si ponga ad integrazione della legalità formale, o delle teorie delle forme procedimentali quali strumenti di tutela dei diritti dei singoli.

In questa prospettiva, allora, il Trattato di Roma - lo si voglia annoverare o meno tra i fenomeni normativi ascrivibili al tipo "costituzione" - rivela innanzi tutto il suo carattere di "documento politicamente ottriato", frutto di elaborazione di un organo che assomiglia molto ai "consigli della Corona" della tradizione liberale ${ }^{16}$. L'ossequio acritico ai suoi testuali contenuti normativi, quindi, rischierebbe di 
trascinare l'interprete "nella tautologia, nella ripetizione circolare di ciò che l'Autorità «ha posto» (positum) come suo comando, magari conquistando a questo l'assenso degli altri"' ${ }^{\prime 1}$. Ciò che sembra a prima vista mancare in tale documento è, infatti, la "rischiosa"18 apertura al pluralismo politico, a quel concetto di democrazia pluralista, cioè, in irriducibile antitesi storica con il positivismo legalistico, aperta ai principi ed ai valori che, oltre il mero schermo della legalità garantiscano al sistema il pieno sostegno della legittimità ${ }^{19}$. Così, ancora, Baldassarre, nel ricondurre all'essenza stessa del fenomeno Costituzione come «legge superiore» l'idea che le libertà ed $\mathrm{i}$ diritti della persona, in quanto superiori alla legge, vengano assunti quale limite e parametro della legittimità della legge stessa ${ }^{20}$, torna a riproporre il problema della democrazia sostanziale quale fonte di legittimazione dei sistemi costituzionali contemporanei. Problema questo che, in relazione ai contenuti della Costituzione europea rimane irrisolto, almeno sotto il profilo della difficoltà di riconoscere l'effettivo valore del rinvio alla legge (ed alle prassi nazionali!) più volte contenuto nella sua Parte II, dedicata alla Carta dei diritti fondamentali, che così sembra concepire tali diritti come subordinati alla legge, piuttosto che come limite essi stessi di rango costituzionale ${ }^{21}$ al legislatore ordinario.

Ritorna la concezione dei diritti fondamentali come diritti pubblici soggettivi, diritti cioè di difesa dalle intrusioni dei pubblici poteri, come libertà negative e non ancora come fonti di legittimazione del potere ${ }^{22}$. In altra sede avevo rappresentato questa esigenza nel costruire il paradigma di legge quale base dell'ordinamento fondandolo sulla effettività del diritto alla partecipazione politica dei destinatari del comando, ripensando in chiave democratica le tesi di Schmitt sul concetto di legge politica $^{23}$. In questo quadro, allora, potrebbe già presentarsi un primo approccio nello studio del problema della effettività dei diritti fondamentali, valutandone la concreta portata in quanto attributi del diritto di partecipazione dei cittadini alla determinazione della politica generale, strumenti degli individui in quanto partecipi della volontà generale, misurando i diritti di libertà sul grado di effettività della partecipazione di tutti alla vita politica, economica e sociale del sistema. Riprendendo ad esempio l'impostazione critica di Floridia a proposito della reale essenza del "governo misto inglese"24 fin dalle sue stesse origini, dalla primigenia funzione di determinazione, da parte dei diritti dei nobili, della volontà comune antagonista a quella del sovrano matura, infatti, la successiva importanza della funzione di sintesi del compromesso 
tra gli interessi di ciascuno nella legge, consentendo di leggere quindi gli stessi diritti di libertà quali diritti politici.

Dall'analisi testuale della Carta dei diritti dell'UE, invece, non emerge oggi né un sistema di situazioni soggettive in funzione di reale limitazione del potere politico, perché i diritti vengono sostanzialmente subordinati, quanto alla concreta definizione del loro contenuto prima ancora che dei loro limiti, alla legge che dovrebbero invece essi stessi limitare ed orientare; né in chiave di costruzione di un ordinamento giuridico fondato sulla rappresentanza, per non dire sugli interessi effettivi dei titolari di quegli stessi diritti. So di ripetermi nel ribadire che ne emerge una configurazione dogmatica dei diritti costituzionali regredita allo stadio delle categorie strutturali proprie dello Stato liberale.

Altro punto, poi, è quello che emerge dalla lettura delle disposizioni c.d. orizzontali della Carta dei diritti ${ }^{25}$, vale a dire quelle che formalizzano l'impianto della disciplina dei diritti sul piano della "interpretazione ed applicazione della Carta" in termini di stretta legalità formale. In primo luogo va segnalato il rilievo dei limiti delle competenze degli Stati membri e delle istituzioni e degli organi dell'Unione conferite dal Trattato medesimo in ogni altra sua parte quale misura della effettiva rilevanza e della possibile garanzia dei diritti medesimi, secondo quanto disposto dall'art. II-111 del Trattato. "Le disposizioni della Carta si applicano alle istituzioni, organi e organismi dell'Unione...come pure agli Stati membri esclusivamente nell'attuazione del diritto dell'Unione. Pertanto i suddetti soggetti rispettano i diritti, osservano i principi e ne promuovono l'applicazione secondo le rispettive competenze e nel rispetto dei limiti delle competenze conferite all'Unione nelle altre parti della Costituzione"26. Se ce ne fosse ancora bisogno, cioè, viene ribadita l'assenza di una specifica competenza dell'Unione europea e degli Stati membri secondo il Trattato in materia di diritti fondamentali...che quindi non incarnano il fondamento del sistema ${ }^{27}$. L'art. II-112 contiene inoltre l'ormai celebre rinvio alla "legge" per la previsione delle "eventuali limitazioni all'esercizio dei diritti e delle libertà riconosciuti dalla...Carta" nel rispetto del "contenuto essenziale di detti diritti e libertà". Dalla disposizione emerge, quindi, uno specifico rilievo attribuito alle "finalità di interesse generale riconosciute dall'Unione". II secondo comma dell'articolo procede poi a sancire la prevalenza di ogni altra disposizione del Trattato in materia di diritti riconosciuti dalla Carta, 
subordinando il loro esercizio "alle condizioni e...limiti ivi definiti", con ciò accentuando il dato funzionalistico o, per lo meno, la sua declinazione normativa. Ma è, soprattutto, nel quinto comma dell'articolo II-112 che si rinviene la disposizione più sintomatica della reale consistenza giuridica dei diritti sotto il profilo del loro contenuto di garanzia. Dopo un nuovo riferimento ai limiti delle competenze delle istituzioni dell'Unione e degli Stati membri in sede di esclusiva attuazione del diritto dell'Unione quale ambito materiale di possibile declinazione normativa delle disposizioni della Carta che contengano dei principi, limitando poi l'efficacia di tali disposizioni nei limiti dei soli contenuti degli atti legislativi ed esecutivi da essi adottati in sede di attuazione; dopo avere quindi ridotto l'efficacia delle disposizioni di principio alla sola ipotesi di una loro espressa attuazione normativa al fine di evitare, almeno nominalmente, possibili fughe in avanti della Corte di giustizia, la norma procede stabilendo che tali disposizioni di principio "possono essere invocate dinanzi ad un giudice solo ai fini dell'interpretazione e del controllo di legalità di detti atti" di attuazione ed esecuzione.

Tra i meriti riconosciuti a Georg Jellinek quale teorico dei diritti pubblici soggettivi in rottura dello schema formale centrato sulla loro derivazione funzionale dai principi storici dello statualismo tedesco si ricorda proprio l'effetto di avere scientificamente giustificato il riconoscimento legislativo del "diritto dei cittadini di ricorrere al giudice amministrativo, contro i comportamenti illegali della pubblica amministrazione direttamente lesivi delle libertà dei singoli”28 quale strumento di concreta garanzia dei diritti medesimi, unica apertura al riconoscimento di un significato sostanziale a tali diritti. Le aperture legislative promosse da Bismarck al fine di dare un concreto contenuto precettivo alle disposizioni in materia di diritti dei sudditi introdotte dalla riforma del 1906 daranno infatti seguito, seppur in misura estremamente contenuta e "con un ritardo di circa un secolo rispetto agli ordinamenti anglosassoni"29 alla concezione dei rapporti tra Stato e sudditi quali in parte condizionati dalla giuridicità dello Stato stesso, annunciando i primi germi di quelle teorie che verranno poi ascritte al filone dello stato liberale di diritto. II principio di legalità formale come primo (e per lungo tempo) unico fondamento giuridico della garanzia dei diritti costituzionali risolto nell'esclusivo strumento del ricorso al giudice amministrativo contro gli atti della pubblica amministrazione contrari alla legge rappresenterà, perciò, la prima vera incrinatura nelle rigide acquisizioni dello 
statualismo tedesco, al quale del resto lo stesso Jellinek rimase sostanzialmente fedele $^{30}$. Come giudicare, allora, le aperture normative ora concesse secondo il tenore delle formule del trattato se non, confermando un'impressione già espressa, come un ritorno all'antico? Quale significato sostanziale potrà attribuirsi oggi nel contesto europeo alla formula "diritti fondamentali"?

II tenore problematico di queste considerazioni richiama del resto le riserve già più volte formulate circa lo scarso valore delle formule ricognitive di tali diritti nella costruzione di una gerarchia delle fonti del diritto europeo che ne garantisca, appunto, il carattere di fondamentalità attribuendo all'atto nominalmente chiamato "legge europea" un ruolo di effettiva trasformazione di tale sistema normativo in funzione della democraticità dell'ordinamento e della centralità dei diritti nei suoi futuri sviluppi ${ }^{32}$.

3. Il ruolo del costituzionalismo per la valorizzazione dei diritti in Europa. Proseguendo nell'analisi credo utile, però, abbandonare per il momento la prospettiva della lettura testuale delle disposizioni del trattato ricognitive della disciplina dei diritti, come accennato integranti il precipitato dello spirito della sovranità degli Stati autori, per il tramite dei loro governi, del Trattato costituzionale. Ciò al fine di formulare una proposta di riflessione animata da altro spirito, lo spirito del costituzionalismo, cercando quindi di leggere il processo di integrazione costituzionale ed i diritti fondamentali magis ut valeant, in attesa del da più parti annunciato avvio della fase di un più accentuato "ruolo costituente" della Corte di giustizia. Qualsiasi testo costituzionale, o documento che politicamente pretenda di essere posto in tale prospettiva, infatti, è suscettibile di interpretazioni e pretese di applicazione anche in contrasto con le c.d. "intenzioni del legislatore" nel tentativo di utilizzare anche una costituzione ottriata quale fonte di produzione di diritto costituzionale sostanziale.

Secondo la prospettiva attuale del possibile sviluppo del diritto europeo e comunitario dei diritti fondamentali l'attenzione va quindi rivolta al modello di integrazione tra gli ordinamenti prodotto della giurisprudenza del Tribunale supremo della nuova entità politica - se questa verrà - la Corte di giustizia matrice della costruzione del nuovo common law europeo. L'analisi non dovrà però, naturalmente, 
più orientarsi alla ricerca dei dati rilevanti sotto il profilo di una ritrovata matrice liberale dei principi dello stato di diritto quanto piuttosto alla ricerca degli elementi per l'affermazione dei valori di garanzia giuridica dei diritti e delle libertà dei cittadini secondo le linee di sviluppo proprie del modello anglosassone di stato di diritto. Almeno a voler prendere seriamente la proposta ricostruttiva, da più parti avanzata, di porre al centro del processo di sviluppo del diritto costituzionale europeo proprio la giurisprudenza creativa, o pretoria che dir si voglia, della Corte di giustizia ${ }^{33}$. La storia stessa dei diritti fondamentali nell'ordinamento europeo è notoriamente frutto della evoluzione della giurisprudenza della Corte di giustizia, fin dagli albori del processo di integrazione. Dall'originaria assenza di un catalogo europeo dei diritti fondamentali ${ }^{34}$ si giunge, infatti, alla loro attuale codificazione proprio per via di consolidamento ed integrazione del sistema di protezione di tali diritti frutto del costante attivismo giurisprudenziale, procedendo alla scrittura della Carta di Nizza proprio per via di sistematizzazione del case-law della Corte di giustizia. Su tali premesse sembra, pertanto, scontato procedere ad un esame comparativo di questo sistema di garanzie con il tradizionale modello di protezione dei diritti costituzionali proprio dei sistemi di common law.

Partendo perciò dalle originarie sistematizzazioni del pensiero costituzionalistico inglese ricordo che Albert Venn Dicey ${ }^{35}$ in tesi generale riteneva che esistessero due modelli di tutela dei diritti sotto il profilo della effettività delle garanzie: "(P)resso di noi i principi generali della costituzione (come per esempio il diritto alla libertà personale, o il diritto di riunione) sono il risultato di decisioni giurisprudenziali che hanno definito i diritti dei privati in particolari casi che sono stati portati alla cognizione delle corti di giustizia, laddove invece in molte forme costituzionali straniere la protezione (...) che è data ai diritti individuali risulta, o sembra risultare, dai principi generali della costituzione".

Ed il modello continentale di costituzionalismo è infatti incentrato sull'articolazione per parti separate tra le disposizioni della costituzione contenenti le formule ricognitive della disciplina dei diritti fondamentali, e quelle dedicate invece alla disciplina dell'organizzazione dei poteri pubblici in funzione di garanzia degli stessi diritti, comprendendo queste la definizione del ruolo politico della legge e la sua stessa struttura procedimentale nonché la declinazione in concreto dei singoli 
istituti di garanzia dei diritti individuali (dall'art. 16 della Dichiarazione dei diritti del cittadino del 1789 alla classica bipartizione professata dalla Costituzione di Weimar). E' così che, a seguire, l'illustre studioso procede con la comparazione dei due modelli di garanzia dei diritti, quello fondato sulle proclamazioni formali proprie delle Carte dei diritti e quello inglese, per il quale gli strumenti di garanzia del diritto individuale ed il diritto individuale stesso figurano legati da un nesso inscindibile, "ubi jus ibi remedium"36. E così è anche per il significato politico-istituzionale del "dominio della legge», primato del diritto ordinario sull'arbitrio del potere; eguaglianza di fronte alla legge di tutti i cittadini, di tutte le classi sociali da cui dedurre il significato sostanziale del concetto di "giustizia»; e, soprattutto, "circostanza di fatto secondo cui...il diritto costituzionale...le regole...configurano non la fonte bensì la conseguenza dei diritti individuali così come essi sono definiti e garantiti dalle corti di giustizia...in tal guisa la costituzione è il prodotto del diritto ordinario del paese" (the common law of the land $)^{37}$.

Di questa sostanziale struttura del modello costituzionale anglosassone, che incentra la disciplina costituzionale dei diritti fondamentali negli stessi strumenti di garanzia in concreto predisposti dall'ordinamento, deducendo quella da questi, si può rendere testimonianza richiamando la stessa dottrina che, anche di recente, ha proceduto ad un'analisi comparata di quel sistema ${ }^{38}$. La disciplina del diritto costituzionale dei diritti fondamentali deve, pertanto, dedursi dal diritto giurisprudenziale, dalla casistica che sulla base delle concrete questioni decise dalle corti comuni consente di costruire una dottrina dei rimedi, come dire, delle effettive garanzie offerte dall'ordinamento a protezione dei diritti dei singoli, contribuendo per via di integrazione a costruire il vero sistema dei diritti costituzionali.

Ora, rispettando la proposta di metodo professata in apertura, abbandonando cioè la tecnica di lettura della disciplina dei diritti fondamentali predisposta dal sistema europeo secondo gli schemi formalistici del positivismo legalistico, quale diritto costituzionale europeo dei diritti fondamentali ci si potrà attendere dalla giurisprudenza delle Corti di Lussemburgo e di Strasburgo soprattutto oggi, a seguito del sostanziale fallimento del maldestro tentativo di costituzionalizzare nominalmente, ad opera di un trattato, la disciplina normativa dei diritti contenuta nella Carta di Nizza? Davvero dovrà accettarsi l'idea che, almeno nel prossimo 
futuro, il diritto europeo dei diritti fondamentali sia costruito per via interpretativa dalle Corti, procedendo dai singoli casi concretamente sottoposti alla loro giurisdizione, per via interpretativa di un materiale normativo costruito per principi generali e regole aperte, indeterminate, fondando le proprie decisioni sulla categorie dell'interpretazione per valori, o per principi. Un rinnovato ruolo creativo, o normativo, che probabilmente abbandonerà i pur timidi tentativi di richiamarsi allo schematismo del rispetto di una fattispecie normativa astratta sì, ma compiuta e dettagliata, verso un dialogo ermeneutico condizionato dalla fattispecie concreta.

4. La comparazione tra le Corti europee e i giudici nazionali: il necessario confronto culturale tra sistemi differenti. Per concludere desidero, infine, porre un'ultima questione, quella della comunicazione tra differenti modelli come necessità culturale per la comparazione tra sistemi diversi di garanzia dei diritti fondamentali ${ }^{39}$ al fine di mutuare, sul piano dell'effettività, gli strumenti più efficaci messi a disposizione dal confronto tra le Corti europee e tra queste ed $\mathrm{i}$ giudici nazionali. Come è noto l'art. I-9, comma 2 , del Trattato costituzionale ipotizza la realizzazione del processo di adesione dell'Unione europea alla Convenzione europea dei diritti dell'uomo. Aggiunge, quindi, che "tale adesione non modifica le competenze dell'Unione definite dalla Costituzione", con ciò ribadendo le già segnalate cautele contro i rischi di un autonomo processo di integrazione costituzionale sul piano dei diritti condotto dalla Corte di giustizia. Ma al di là dei limiti posti dai Governi degli Stati membri, come dire, "in sede costituente", quali potrebbero essere gli effetti, o almeno alcuni tra questi, di un'adesione formale dell'Unione europea al Trattato di Roma del 1950 ? E, soprattutto, quali problemi potrebbero ipotizzarsi nel confronto tra le due Corti europee alla luce dei vincoli che ne deriverebbero dalla Convenzione europea per le stesse Istituzioni UE, malgrado il richiamato ostacolo ad un'espansione, in termini di garanzia dei diritti, delle competenze di queste ultime sul piano dell'integrazione costituzionale?

La prima constatazione a meritare la nostra attenzione attiene all'abitudine della Corte di giustizia di ricostruire come principi del diritto comunitario dei diritti fondamentali la stessa giurisprudenza della Corte di Strasburgo, con ciò procedendo alla tradizionale rivisitazione delle loro disciplina in chiave funzionale agli sviluppi del processo di integrazione comunitaria ${ }^{40}$. A ciò deve, peraltro, aggiungersi la 
considerazione che la Corte di Strasburgo finirebbe reciprocamente col giudicare sulle violazioni della Convenzione europea perpetrate dalle istituzioni UE, ivi compresa la Corte di Lussemburgo. Certo sulle modalità e gli effetti dei vincoli deducibili per quest'ultima dalla giurisprudenza CEDU molto dovrebbe essere deciso in sede di stipula dell'accordo sulle modalità dell'adesione alla CEDU da parte dell'UE, riflessione problematica su cui si sofferma nel dettaglio la citata analisi di Panunzio ${ }^{41}$, ma non può non pesare nella vicenda anche la circostanza per cui la casistica prodotta dalla lunga e pervasiva esperienza della Corte europea, rinvenibile nelle molte raccolte e ricostruzioni critiche formulate dalla dottrina in questi anni, riesce ad essere ordinata a sistema soltanto con molte difficoltà e semplificazioni. La giurisprudenza CEDU, infatti, provocata su iniziativa dei singoli individui è spesso condizionata da questa matrice, per così dire, casistica che pur essendo ad un tempo la causa vera della sua forza espansiva rende di fatto non agevole la pretesa di ricostruire sulla sua base una concreta disciplina dei diritti e delle libertà secondo la prassi applicativa della Convenzione. Sul piano concreto, quindi, la Corte di Giustizia avrà a sua disposizione non pochi margini interpretativi a sostegno della sua tradizionale capacità creativa, cosa che potrà contribuire a realizzare una propria prassi casistica da contrapporre, se del caso, alla giurisprudenza CEDU. Tanto più se si osserva, con Panunzio, che "il ruolo della Corte di Strasburgo è più «esteso", mentre quello della Corte di Lussemburgo è più «penetrante ${ }^{42}$, grazie alla più forte capacità giuridica della sua giurisprudenza di imporsi negli ordinamenti degli Stati membri con efficacia diretta.

Ma quale sarà, piuttosto, l'effettivo ruolo della Corte UE? A differenza della Corte di Strasburgo, infatti, chiamata ad intervenire nel caso concreto al fine di ristabilire l'ordine del minimo standard di protezione violato dallo Stato membro come dire, in funzione di protezione esterna, l'ordinamento UE e, per esso, la stessa Corte di Lussemburgo dovrebbero assumere un ruolo positivo facendosi carico, al pari degli Stati costituzionali, di una politica di implementazione dei diritti fondamentali ${ }^{43}$. Con riferimento alla necessità di procedere ad un incremento, nei processi di integrazione, dei livelli di protezione dei diritti fondamentali superando la riduttiva dottrina del minimum standard, lo stesso Panunzio conclude la sua analisi con l'auspicio che la UE si assuma "anche un ruolo «positivo»: quello...di portare avanti una politica mirante alla realizzazione ed implementazione di quei diritti, anche 
attraverso l'azione degli Stati" ${ }^{44}$. Auspicio che rappresenta, ad un tempo, una prima importante conclusione critica della proposta di metodo dell'illustre studioso, tesa a misurare il grado sostanziale di protezione dei diritti fondamentali nel sistema europeo, alla ricerca di una dottrina costituzionale che imponga a tale sistema di professare una teoria dei diritti che siano fondamento, e non più solo limite, delle sue linee di sviluppo politico, una teoria che attribuisca a tali diritti il ruolo di fondare l'ordinamento e non soltanto, come ora sembra, di indurre le istituzioni e gli Stati ad un atteggiamento di distaccato rispetto.

Sul piano pratico, della effettività della tutela, due sono allora le possibili prospettive di analisi che si potrebbero suggerire in attesa dei primi sviluppi giurisprudenziali nelle more - ed in assenza - della ratifica del Trattato costituzionale. Da un lato la ricerca del significato giuridico dell'inserimento nella Carta dei diritti fondamentali di situazioni giuridiche garantite in ambiti materiali privi di competenze per le istituzioni UE nelle quali, pertanto, secondo lo stessa Trattato non dovrebbero potersi espandere gli interventi normativi comunitari e dell'UE; ed un'attenta riflessione, all'opposto, sul significato politico dei casi di più forte integrazione costituzionale che, seppur occasionalmente, la giurisprudenza delle Corti di Strasburgo e di Lussemburgo sono riuscite a garantire rispetto agli interventi svolti, nelle stesse materie, dalle Corti costituzionali nazionali45. Una prospettiva di riflessione teorica, quindi, ed un'analisi, per così dire, sul campo che consentano di svelare almeno i primi elementi di queste evidenti incongruenze emergenti dal confronto tra i testi normativi e la reale consistenza del livello di protezione dei diritti fondamentali nel sistema europeo.

Ma un ulteriore elemento merita forse qualche accenno di attenzione una volta che si sia scelta la strada di procedere per la via del confronto tra i diversi sistemi, starei per dire "livelli", di garanzia dei diritti nel processo di integrazione europea. E' il problema, al quale pure si è già fatto qualche riferimento, della reale adattabilità del sistema nazionale di protezione dei diritti fondamentali alla rivoluzione culturale imposta dall'ingresso delle categorie del common law nel diritto comune europeo dei diritti fondamentali. Per un esempio evidente di questa tensione basti il rinvio alla giurisprudenza europea in materia di due process of law che per il tramite della Convenzione europea dei diritti dell'uomo, degli artt. 5 e 6 di tale Trattato ${ }^{46}$, esercita 
da tempo una fortissima pressione sugli ordinamenti nazionali di diritto continentale tuttora refrattari a lasciarsi permeare da un'effettiva declinazione del right to hearing. Per l'ordinamento italiano basti il rinvio a due recenti episodi per lasciare intendere in quale prospettiva vada affrontato il problema qui posto: l'art. 21-octies della legge $n$. 241 del 1990, introdotto dalla riforma del 20057; e la sentenza del Consiglio di Stato n. 1559 del $2004^{48}$.

La legge di riforma della disciplina del procedimento amministrativo contiene, infatti, una disposizione ${ }^{49}$ che nella prospettiva di proteggere le scelte compiute dall'amministrazione nel provvedimento finale dai vizi di mera forma pretestuosamente dedotti in giudizio, al fine ultimo di salvaguardare, con il provvedimento, l'interesse generale rischia però di compromettere quanto di più significativo si è inteso affidare al procedimento amministrativo, la garanzia di un sano e corretto contraddittorio. Naturalmente al momento è prematuro prevedere quale orientamento giurisprudenziale verrà assunto quale dominante ${ }^{50}$ nel dare attuazione a questa nuova regola, se prevarrà cioè veramente la difesa dell'interesse pubblico contro i tentativi di utilizzo degli strumenti procedimentali in chiave meramente formalistica a scapito della giustizia sostanziale o se, all'inverso, si svolgerà a protezione degli interessi forti dedotti e difesi nel procedimento anche a costo del sacrificio degli interessi dei soggetti deboli scardinando gli ultimi strumenti a tutela dei diritti dei singoli per un'effettiva partecipazione al procedimento. Ma anche la sola ipotizzabilità di questa seconda linea interpretativa è sufficiente motivo per richiamare l'attenzione sull'importanza dei vincoli culturali del giudice nella declinazione delle garanzie in funzione della loro concreta effettività. Nell'ottica comunitaria ed europea, infatti, la garanzia del due process of law non ammette interpretazioni riduttive, deducendosi dalla mera violazione di questa regola l'illegittimità del provvedimento.

La sentenza del Consiglio di Stato, invece, decide una questione che ha visto contrapporsi un comune, quale ente esponenziale degli interessi politici riferibili ad una frazione del territorio e la Regione Puglia in relazione all'approvazione, da parte di quest'ultima, del nuovo piano sanitario con previsione della disattivazione di alcune unità ospedaliere di cui una servente il comune ricorrente. Per proteggere la delibera assunta la Regione, nelle more del giudizio di I grado, aveva provveduto ad 
approvare una legge-provvedimento di analogo contenuto ottenendo così dal TAR Puglia una decisione di improcedibilità. Contestando la "legificazione dell'atto amministrativo" nel corso dello stesso processo quale espediente lesivo del diritto ad un'effettiva tutela giurisdizionale il comune ricorrente chiedeva al Consiglio di Stato di sollevare una questione di legittimità costituzionale per violazione degli artt. 24 e 113 Cost. o la disapplicazione della legge regionale per violazione degli artt. 6 e 13 della Convenzione europea dei diritti dell'uomo e sulla base della diretta applicabilità necessaria del conforme principio comunitario del diritto ad un'effettiva tutela giurisdizionale. II giudice amministrativo, nel rigettare il ricorso, motiva osservando che il diritto della CEDU, attuata in Italia con un ordine di esecuzione adottato con legge ordinaria, di per sé non può fungere da parametro di costituzionalità di una legge interna; è in teoria abrogabile ad opera di una legge successiva sulla base del criterio della successione delle leggi nel tempo e non consente la disapplicazione, ad opera del giudice, del diritto interno con esso incompatibile. Sul fronte del diritto comunitario in materia di diritti fondamentali, pure invocato dal ricorrente, e del diritto ad un'effettiva tutela giurisdizionale in particolare, il Consiglio di Stato ritiene che nel caso di specie esso non possa considerarsi vincolante in virtù della sua applicabilità con esclusivo riferimento alla tutela giurisdizionale dei soli diritti di derivazione comunitaria ${ }^{51}$. Anche l'eccezione di costituzionalità viene quindi rigettata.

I due episodi richiamati testimoniano la difficoltà culturale di coniugare il giudizio sulla legalità di un atto, o altra decisione pubblica rispetto alle categorie proprie di un sistema che tale legalità non deduca sulla base di un previo paradigma formale, una norma generale ed astratta, bensì in relazione alla concreta declinazione delle regole del contraddittorio, del diritto alla effettiva partecipazione al procedimento ${ }^{52}$, o al processo, in funzione della tutela dei diritti e degli interessi del singolo, pretendendo così di ricostruire il concetto di legalità sulla base del diverso paradigma del due process of law. Più in particolare la sentenza del Consiglio di Stato, criticabile anche sotto altri profili, soprattutto in materia di rapporti tra le fonti internazionali pattizie ed il diritto interno, dimostra come la concezione della legalità e del diritto alla tutela giurisdizionale nel sistema italiano si fondi su una visione formale del rapporto tra atti amministrativi, leggi e diritti individuali poco attenta al diverso impianto concettuale che vede nella effettiva partecipazione del singolo al procedimento (o al processo) e nella efficacia degli strumenti legali a sua disposizione l'essenza stessa della 
garanzia dei diritti sostanziali. Non sarà, del resto, un caso se il Parlamento italiano sia stato costretto a procedere alla revisione testuale dell'art. 111 della Costituzione per ottenere dalla giurisprudenza della stessa Corte costituzionale il rispetto del paradigma partecipativo quale strumento essenziale del principio del contraddittorio. La nuova formulazione della disposizione costituzionale contiene, solo ora ${ }^{53}$, infatti, l'espresso richiamo del principio "del giusto processo regolato dalla legge", del "contraddittorio tra le parti, in condizioni di parità", della "ragionevole durata" del processo, oltre alla declinazione nel dettaglio delle regole processuali penali in materia di acquisizione delle prove e di effettività del contraddittorio nella formazione stessa della prova. Alcune di queste regole, che la dottrina già riteneva incluse nel sistema costituzionale di garanzie processuali ${ }^{54}$, soprattutto in materia penale, ridotte nella legislazione ordinaria avevano invece ottenuto una radicale censura da parte del giudice di legittimità delle leggi ${ }^{55}$, come dire, meno sensibile alla declinazione secondo la cultura anglosassone ${ }^{56}$ di tali strumenti di garanzia.

E così torniamo al punto di partenza, al confronto tra le categorie culturali della effettività della tutela dei diritti declinate secondo i paradigmi dei sistemi di common law, ai quali di fatto si ispirano la giurisprudenza comunitaria ed europea in materia di tutela dei diritti fondamentali, e quelle proprie dei sistemi, come quello italiano, fondati sulla prevalenza di un formale parametro legale previo e generale che contenga la disciplina dei diritti e delle relative garanzie. In realtà la rule of law è un elemento fondamentale del processo di integrazione costituzionale condotto in Europa dalle Corti di Lussemburgo e di Strasburgo ${ }^{57}$. Nell'ambito del diritto comunitario, così, tra le conseguenze di sistema deducibili dalla lettura degli artt. 220 ss. in materia di diritti alla tutela giurisdizionale ${ }^{58}$ dei singoli vanno innanzi tutto annoverati la pretesa alla effettività dei rimedi giurisdizionali; la loro completezza in termini di strumenti di garanzia disponibili; e la essenzialità del meccanismo del rinvio pregiudiziale ex art. 234 quale mezzo di salvaguardia della prevalenza sul diritto nazionale, con il diritto comunitario direttamente applicabile, dei principi della rule of law. In ciò si sostanzia il principio di legalità nel sistema UE, nella pretesa alla diretta applicabilità del diritto comunitario e nella prevalenza delle sue regole sulle norme del diritto interno con esse incompatibili. E sul piano della effettività dei diritti, vale a dire sul piano delle garanzie, la giurisprudenza della Corte di Giustizia ha da tempo proceduto a ricostruire nel dettaglio gli elementi in cui queste devono riconoscersi. 
Così è per il principio del diritto ad un'effettiva tutela cautelare ${ }^{59}$; o per l'affermazione del diritto alla risarcibilità dei danni prodotti dal cattivo esercizio della funzione legislativa; o ancora per la declinazione dei caratteri che ogni ordinamento nazionale deve necessariamente riconoscere e garantire al giudice interno ed alla funzione giurisdizionale perché essi possano predicarsi del ruolo di giudici comunitari, sebbene giudici nazionali, nell'applicazione ed attuazione diretta del diritto comunitario stesso ${ }^{60}$.

L'attuale pretesa storico-politica di ottenere l'affermazione in Europa di un sistema costituzionale di tutela dei diritti fondamentali fondato sull'effettività degli strumenti di garanzia, quindi, deve opporre al concetto di legalità professato dalla Corte di Giustizia in funzione della prevalenza del diritto comunitario un concetto di legalità costruito intorno ad un sistema di rimedi giudiziali e procedimentali a protezione di tali diritti dei cittadini. Così come in Inghilterra, nella lettura di Dicey, "l'attore fondamentale del processo costituzionale sono state le Corti" che hanno "operato un vero e proprio processo di costituzionalizzazione dei diritti garantiti dal common la $w^{61}$, nel sistema europeo e comunitario il ruolo creativo della giurisprudenza nel campo dei diritti e delle libertà dovrebbe indurre ad un radicale mutamento di prospettiva culturale gli interpreti e gli attori politici di quel sistema, per l'affermazione anche in esso di una dinamica fattuale di affermazione dei principi del costituzionalismo connettendo "in maniera inseparabile gli strumenti di tutela di un diritto e il diritto da tutelare"62. La legalità dell'ordinamento dato deriverebbe, allora, non più dalla verifica del formalistico rispetto di regole astratte declinanti la disciplina dei diritti e delle libertà, inapplicabili in assenza di un sistema fondato sul principio della divisione dei poteri che conosca, pertanto, un'effettiva funzione legislativa predicabile in senso sostanziale, bensì dal consolidamento di un'effettiva prassi giurisprudenziale di garanzia quotidiana di tali diritti che ne determini la reale consistenza in ogni ipotesi condotta all'attenzione di un giudice.

I riflessi sul piano interno di questa pretesa rivoluzione culturale sarebbero immediati, e di immediata evidenza. II tentativo perpetuo di un cittadino comune di ottenere giustizia contro la pervicace ostinazione di un sistema legalistico che consente di opporre un preteso difetto di giurisdizione in ogni grado del giudizio di merito non riuscirebbe, infatti, ad ottenere udienza presso i giudici, né tollererebbe - 
come in casi analoghi ha già non tollerato ${ }^{63}$ - che la Corte costituzionale si pronunci nel merito per la seconda - e ultima? - volta nel corso dello stesso giudizio su tale pretesa $^{64}$.

Del resto non può negarsi oggi un più generale momento di confusione culturale frutto dell'attuale disordine giurisprudenziale che in Italia sembra essere conseguenza della rottura del paradigma della legalità formale in totale assenza di un contrapposto rigore di sistema nel costringere la stessa giurisprudenza a compensare tale carenza con un costruttivo rispetto del vincolo del precedente che contribuirebbe al formarsi di un case law di stabilizzazione dei diversi orientamenti interpretativi. La perdita di effettività delle categorie del previo parametro formale, infatti, genera oggi un incoerente sviluppo giurisprudenziale che su molte questioni paga lo scotto di un approccio meramente occasionale ai criteri di una più ampia discrezionalità dei giudici. Sostenuti dalle dinamiche delle teoriche multilevel questi coltivano, infatti, il rischio inconsapevole di un'alterata commistione di regole appartenenti a culture giuridiche diverse perdendo la capacità critica di agire in compensazione, comparando tali elementi in funzione di salvaguardia della unità e della stabilità del sistema. Questo generalizzato atteggiamento, nel funzionamento dell'ordinamento, diviene però difetto strutturale di sistema che manda a rischio la stessa funzione di nomofilachia, non compensata dal comporsi di un common law che ne surroghi le prestazioni di stabilità ed unità a favore dell'ordinamento medesimo.

\section{OS DIREITOS FUNDAMENTAIS E SUA EFETIVIDADE}

\section{RESUMO}

Neste ensaio eu entendo aproximar o debate sobre o tema dos direitos fundamentais, envolvendo a perspectiva de sua efetividade principalmente no sistema constitucional europeu. Portanto, parte-se da aprofundada diferença que existe entre os membros estatais e os múltiplos aspectos culturais e sociais da cidadania européia.

Palavras-chave: Constituição. Direitos Fundamentais. Sociedade Européia. 


\section{NOTAS}

1 Professor da Universidade de Chieti-Pescara e Roma-Trè, Itália. Professor convidado da Universidade de Santa Cruz do Sul, RS, Brasil.

2 Perché l'espressione non sia causa di equivoci chiarisco da subito l'intenzione di lasciare impregiudicata in questa sede ogni considerazione circa la natura formalmente costituzionale dell'attuale versione dei trattati comunitari guardando piuttosto alla necessità di procedere comparativamente ad un serrato confronto di tale fenomeno con i sistemi costituzionali nazionali, anche soltanto riferendo delle implicazioni costituzionali deducibili dallo stesso dato di integrazione materiale tra gli ordinamenti. Sul piano dei problemi di metodo rinvio a titolo di esempio, anche solo al fine di condividerne la consapevole attenzione alle complessità di fondo, alle differenti impostazioni di C. Pinelli, II momento della scrittura. Contributo al dibattito sulla Costituzione europea, Bologna, 2002, spec. 19 ss., 45 ss., 59 ss., 183 ss.; Id., La Carta europea dei diritti e il processo di "costituzionalizzazione" del diritto europeo, in Aa.Vv., Riflessi della Carta europea dei diritti sulla giustizia e la giurisprudenza costituzionale: Italia e Spagna a confronto, Milano, 2003, 55 ss.; G. Guzzetta, Declinazioni del dualismo comunitario: dall'aut aut all'et et, in Id. (a cura di), Questioni costituzionali del governo europeo, Padova, 2003, 5 ss.; S. Mangiameli, La forma di governo europea, ivi, 67 ss.; A. Ruggeri, Quale costituzione per l'Europa?, in Anuario Iberoamericano de Justicia Constitucional, 8/2004, 455 ss.; F. Mancini, fin dalla Introduzione a Id., Democrazia e costituzionalismo nell'Unione europea, Bologna, 2004, 20 s., 33 ss., ed al bel libro di F. Palermo, La forma di Stato dell'Unione europea. Per una teoria costituzionale dell'integrazione sopranazionale, Padova, 2005. Si vedano altresì, ora, almeno le relazioni di M. Luciani, Gli atti comunitari e $i$ loro effetti sullintegrazione europea, e M. Cartabia, I rapporti tra le istituzioni europee e i parlamenti nazionali: che cosa resta e che cosa si perde in caso di mancata ratifica, e l'intervento di G. Amato al convegno annuale dell'AIC su L'integrazione dei sistemi costituzionali europeo e nazionali, Catania, 14-15 ottobre 2005, in corso di stampa ma disponibili sul sito dell'AIC medesima. Ma sul punto, nuovamente, infra.

3 Firmato a Roma il 29 ottobre 2004.

4 Anche qui chiarendo da subito che utilizzo questa espressione come semplice sintsi verbale per non ripetere ogni volta "Trattato che istituisce una Costituzione per l'Europa", essendo tra quanti ha in più sedi criticato proprio questa confusione terminologica, frutto cioè del pericoloso abuso del concetto di "costituzione europea".

5 Per comodità rinvio alle rassegne ed agli studi che di recente hanno già provveduto a verificare il tasso di effettività delle disposizioni sui diritti contenute nella Carta di Nizza. Tra le altre si vedano, così, i lavori di A. Celotto, G. Pistorio, L'efficacia giuridica dellaCarta dei diritti fondamentali dell'Unione europea (rassegna giurisprudenziale 2001-2004), in www.associazionedeicostituzionalisti.it ma in corso di pubblicazione in Giur.it., 2005; R. Calvano, La Corte d'Appello di Roma applica la Carta dei diritti Ue. Diritto pretorio o Jus comune europeo?, in www.associazionedeicostituzionalisti.it; Id. (a cura di), sentt. in cause T-54/99 del 30 gennaio 2002, e T-377/00, T-379/00, T-380/00, T-260/01, T-272/01 del 15 gennaio 2003, ibidem. Della stessa autrice si veda già, peraltro, la più amplia riflessione condotta ne La Corte di Giustizia e la Costituzione europea, Padova, 2004, spec. 232 ss.

6 Per una emblematica panoramica dei complessi spunti di riflessione teorica di tale approccio di metodo rinvio senz'altro alla ricca messe di contributi raccolti nel volume a cura di P. Bilancia e E. De Marco, La tutela multilivello dei diritti. Punti di crisi, problemi aperti, momenti di stabilizzazione, Milano, 2004, con un'indagine aperta anche alle dimensioni substatali di protezione dei diritti. Si v., comunque, già I. Pernice, Multilevel Constitutionalism and the Treaty of Amsterdam: European Constitution-making rivisited?, in Comm.Mar.Law.Rev., 1999, 703 ss.

7 Desidero precisare fin da subito che le ipotesi ricostruttive del sistema dei diritti fondamentali nel contesto europeo sono qui rielaborate in chiave meramente teorica al fine di utilizzare questa semplificazione per chiarire l'approccio di metodo perseguito, evitando così di dover richiamare gli studi dedicati al tema riconducibili a ciascun filone di pensiero, cosa che si renderebbe impossibile senza dover poi chiarire nel dettaglio le molte importanti implicazioni teoriche qui necessariamente pretermesse per le ragioni spiegate.

8 Per semplicità rinvio al mio / diritti fondamentali come conquiste sovrastatali di civiltà. I/ diritto di proprietà nella CEDU, Torino, 2002. 
9 Basti scorrere l'elenco delle fattispecie normative ricognitive dei diritti negli artt. II-62 ss. del Trattato costituzionale, per un primo commento dei quali non posso non rinviare almeno a $\mathrm{R}$. Bifulco, M. Cartabia, A. Celotto (a cura di), l'Europa dei diritti. Commento alla Carta dei diritti fondamentali dell'Unione europea, Bologna, 2001; G.F. Ferrari (a cura di), I diritti fondamentali dopo la Carta di Nizza. Il costituzionalismo dei diritti, Milano, 2001; L. Ferrari Bravo, F.M. Di Maio, A. Rizzo, Carta dei diritti fondamentali dell'Unione europea, Milano, 2001.

10 n causa C-117/01, sentenza del 7 gennaio 2004.

11 In causa C-112/00, sentenza del 12 giugno 2003.

12 Per semplicità rinvio alle proposte di analisi di A. Pace, $A$ che serve la Carta dei diritti fondamentali dell'Unione europea? Appunti preliminari, in Giur. cost., 2001, 193 ss.; U. De Siervo, L'ambigua redazione della Carta dei diritti fondamentali nel processo di costituzionalizzazione dell'Unione europea, in Dir.pubbl., 2001, 33 ss.; G. Ferrara, Intervento, in Le libertà e i diritti nella prospettiva europea, Atti della giornata di studio in memoria di Paolo Barile, Padova, 2002, 143 ss., ma già da prima in Id., Da Weimar a Maastricht, in la rivista del manifesto, n. 10/2000, 24 ss.; G. Azzariti, II valore della Carta dei diritti fondamentali nella prospettiva della costruzione europea: dall'Europa dei mercanti all'Europa dei diritti?, in S. Labriola (a cura di), Ripensare lo Stato, Milano, 2003, 393 ss.; P.F. Grossi, Alcuni interrogativi sulle libertà civili nella formulazione della Carta di Nizza, in F. Fernández Segado (Ed.), The Spanish Constitution in the European constitutional context, Madrid, 2003, 279 ss., spec. 312 ss. Ma si veda altresì l'opposta ricostruzione critica formulata ad esempio da A. Barbera, La Carta europea dei diritti e la Costituzione italiana, in Le libertà e i diritti, cit., 107 ss. Sintomatica di tale fenomeno è, infine, la prospettiva di analisi suggerita da G. Di Plinio, La Carta dei diritti nel processo di integrazione europea, in G.F. Ferrari (a cura di), I diritti fondamentali dopo la carta di Nizza. Il costituzionalismo dei diritti, Milano, 2001, 145 ss., spec. 151 s.; Id.; La costituzione economica nel processo costituente europeo, in Dir.pubbl.comp.eu., 2003, 1786 ss., il quale rilegge la stessa Carta dei diritti in chiave funzionale alla vigente costituzione economia europea (dall'autore intesa in senso materiale), essendo essa "già orientata al mercato", per cui l'interpretazione che ne darà la Corte di Giustizia non potrà tradire "la missione funzionalista dell'Unione". Sembra di capire che la funzione politica della Carta dei diritti risieda, secondo tale studioso, proprio nell'intento di neutralizzare la portata politica dei principi a loro fondamento quale strumento in possesso dei singoli Stati membri per sostenere scelte di "dumping costituzionale". Un catalogo comune dei diritti fondamentali, quindi, quale riduzione dell'autonomia costituzionale degli Stati membri a tutela del mercato unico, altrimenti minacciato dalle asimmetrie statuali nel livello di garanzia degli stessi diritti costituzionali.

13 G. Silvestri, La tutela dei diritti fondamentali nell'Unione europea dopo la Carta di Nizza, in Studi in onore di Gianni Ferrara, III, Torino, 2005, 610 ss., nel ricordare come in materia di diritti fondamentali le Corti abbiano ormai da tempo "abbandonato gli schemi consunti del positivismo legalistico", riflette sulla probabilità che la Corte di Giustizia utilizzi documenti quali la Carta al fine di elaborare in via giurisprudenziale fattispecie ricognitive dei diritti da applicare nel caso concreto.

14 Si veda quanto sostenuto in proposito da P. Ridola, La Carta dei diritti fondamentali dell'Unione europea e le «tradizioni costituzionali comuni» degli Stati membri, in S. Panunzio, E. Sciso (a cura di), Le riforme istituzionali e la partecipazione dell'Italia all'Unione europea, Milano, 2002, 83 ss., spec. 101, 107. L'illustre autore aveva già segnalato il rilievo dato dalle Costituzioni del secondo dopoguerra "al profilo della effettività dei diritti costituzionali", richiamando l'aspirazione alla "libertà dal bisogno" nel saggio Libertà e diritti nello sviluppo storico del costituzionalismo, in R. Nania, P. Ridola (a cura di), I diritti costituzionali, I, Torino, 2001, 39 s.

15 Ma di recente si veda anche l'approfondita ricostruzione critica proposta da F. Salmoni, Diritti sociali e Unione europea. Dall'ordinamento comunitario allo stato sociale europeo, in Studi Ferrara, cit., III, 531 ss. Per un informato giudizio circa i difetti strutturali della c.d. "Europa sociale" v., invece, G. Garavini, I sommersi e i salvati. Emarginazione, politica sociale e diritti nella storia dell'integrazione europea, in L. Barca, M. Franzini (a cura di), Legittimare l'Europa. Diritti sociali e crescita economica, Bologna, 2005, 107 ss.

16 Per citare le più forti espressioni di S. D'Albergo, Diritto e stato tra scienza giuridica e marxismo, Roma, 2004, 339 s., 342 ss., 348, a segnalare la regressione in atto verso i principi della democrazia formale dello stato liberale di diritto ed il contestuale superamento del "costituzionalismo democratico-sociale" del secondo Dopoguerra.

17 A. Baldassarre, Miseria del positivismo giuridico, in Studi Ferrara, cit., I, 208, nel ricostruire l'essenza del positivismo liberale in assenza di costituzionalismo. Ma sul punto si veda ancora, infra, nel testo.

18 per gli Stati europei, Signori dei trattati e redattori costituenti del testo del Trattato. 
19 Ho cercato di riferire su questa prospettiva di analisi, seppur polemicamente, nel mio recente Referendum, populismo e moneta unica. A proposito della costituzione europea, in http://www.costituzionalismo.it/articolo.asp?id=182, fasc. n. 3/2005, al quale rinvio semplicemente per non ripetermi.

20 Op.ult.cit., 220 ss.

21 Riprendo qui la prospettiva problematica già assunta in Considerazioni critiche sul concetto di "legge europea”, in Aa.Vv., In memoria di Giovanni Motzo, Napoli, 2004, 51 ss. Ma si veda, altresì, la critica a tale impostazione formulata da F. Sorrentino, Relazione al Convegno AIC su Separazione dei poteri e funzione giurisdizionale, Padova, 22-23 ottobre 2004. Dello stesso autore sul punto già Brevi riflessioni sui valori e sui fini dell'Unione nel progetto di costituzione europea, in Dir.pubbl., 2003, 815 s. Per una diversa lettura critica, alla ricerca di una "nozione funzionale di legislatore" europeo seguendo un approccio di metodo che qui non è possibile discutere per ragioni di spazio si v., inoltre, S. Niccolai, Tra Costituzione e amministrazione: la produzione del diritto in ambito comunitario, in I/ Filangieri, 2004, 555 ss., spec. 584 ss.

22 Rinvio qui, ancora, al mio / diritti fondamentali, cit., spec. 13 ss.

$23 \mathrm{~V}$. la ricostruzione problematica in Sul concetto di legge politica: una prospettiva di analisi, in $\mathrm{F}$. Modugno (a cura di), Trasformazioni della funzione legislativa, Giuffrè, Milano, 1999, 195 ss.

24 II riferimento immediato va allo scritto Fortuna e crisi del governo misto nella costituzione inglese, in Materiali per una storia della cultura giuridica, XXX, 2000, 305 ss. Per la ricostruzione di tale percorso critico, più nel dettaglio rinvio al mio / diritti fondamentali, cit., spec. 12 ss.

25 Mi riferisco alle disposizioni contenute negli artt. II-111 ss. del Trattato costituzionale.

26 Corsivi miei.

$27 \mathrm{~V}$., sul problema delle competenze e per una conseguente ricostruzione problematica della teoria dei c.d. controlimiti, le interessanti riflessioni formulate da M. Cartabia, "Unità nella diversità": il rapporto tra la costituzione europea e le costituzioni nazionali, in Fondazione Cesifin, "Giornata di studio in ricordo di Alberto Predieri. Sul trattato che istituisce una costituzione per l'Unione europea", Firenze, 18 febbraio, 2005, in corso di pubblicazione.

28 Come ricorda A. Baldassarre, nella voce Diritti pubblici soggettivi, in Enc.giur.Treccani, XI, $1989,4$.

29 Ancora A. Baldassarre, op.ult.cit., 5.

30 Si veda ancora quanto riferito da A. Baldassarre, op.ult.cit., 4 ss., nonché da A. Pace, Problematica delle libertà costituzionali. Parte generale, Padova, 2003, 66 ss.

31 Si veda, infatti, quanto osserva A. Pace, La dichiarazione di Laeken e il processo costituente europeo, in Riv.trim.dir.pubbl., 2002, 636 ss. a proposito del nesso inscindibile tra democraticità dell'ordinamento europeo e tenore normativo delle disposizioni sui diritti e le libertà dei cittadini. Sul problema della democraticità del sistema comunitario si vedano, ora, le diverse conclusioni dedotte rispettivamente da F. Capelli, II sistema istituzionale dell'Unione europea come fondamento di una nuova forma di democrazia, in Dir.com.sc.int., 2004, 221 ss. e da G.F. Mancini, Per uno Stato europeo, ora in Id., Democrazia e costituzionalismo, cit., 99 ss., spec. 116 ss.

32 Senza richiamare per l'ennesima volta i miei precedenti lavori mi confortano ora nell'analisi le considerazioni formulate da G. Amato, nel saggio dall'emblematico titolo In Europa, finalmente, Montesquieu e Cammeo, in Studi Ferrara, I, 121 ss., spec. 127 s. a proposito della necessità di ricondurre gli atti normativi europei "alle distinzioni e alla gerarchia della separazione dei poteri" al fine di individuare quelli tra essi che "hanno indole legislativa", e circa "la generale inconsapevolezza della dottrina, che ha seguito negli anni la vicenda comunitaria, descrivendola così come si veniva dipanando, ma mai cogliendo i germi di un tale problema. In tutti i libri che ho letto ho visto descritti gli atti, descritte le procedure, ma non ho trovato nulla che andasse al di là della evoluzione degli epifenomeni. Ben più utile è stato tornare alla letteratura di oltre un secolo fa...", corsivi miei. Ma si vedano altresì le considerazioni già formulate da G.G. Floridia (con la collaborazione di L.G. Sciannella), Il cantiere della nuova Europa. Tecnica e politica nei lavori della Convenzione europea, Bologna, 2003, 149, “...sicché la «legge europea» diventerebbe” (dovrebbe diventare!) "- ove trovasse l'appoggio di forme capaci di legittimarla con la pienezza rappresentativa e decisionale di un adeguato corpo legislativo - la fonte portatrice dei caratteri e del ruolo tradizionalmente ascritto alla legge negli ordinamenti costituzionali in genere, e alla legge federale nei sistemi federali”, corsivo mio.

33 E' la lezione di metodo da ultimo esemplarmente proposta da S. P. Panunzio, I diritti fondamentali e le Corti in Europa, in Id. (a cura di), I diritti fondamentali e le Corti in Europa, Napoli, 2005, 3 ss. Ma si vedano già, ad es., G. Zagrebelsky, Corti europee e corti nazionali, in S.P. Panunzio (a cura di), I costituzionalisti e l'Europa. Riflessioni sui mutamenti costituzionali nel processo d'integrazione europea, Milano, 2002, 531 ss.; A. Pizzorusso, II patrimonio costituzionale europeo, Bologna, 2002, spec. 17 ss., 172 ss.; R. Toniatti, Il principio di rule of law e la formazione giurisprudenziale 
del diritto costituzionale dell'Unione europea, in S. Gambino (a cura di), Costituzione italiana e diritto comunitario. Principi e tradizioni costituzionali comuni. La formazione giurisprudenziale del diritto costituzionale europeo, Milano, 2002, 503 ss.; A. Ruggeri, Carta europea dei diritti e integrazione interordinamentale, dal punto di vista della giustizia e della giurisprudenza costituzionale (notazioni introduttive), in Aa.Vv., Riflessi della Carta europea dei diritti, cit., 7 ss.; E. Rossi, La carta europea dei diritti e l'espansione del "diritto giurisprudenziale", ivi, 289 ss. Criticamente, ancora, A. Pace, La dichiarazione di Laeken, cit., spec. 642 ss.; Id., A che serve la Carta europea, cit. nonché, in una più ampia prospettiva di analisi in Id., Le sfide del costituzionalismo nel XXI secolo, in Dir.pubbl., 2003, spec. 896 ss.

${ }^{34}$ Non posso non rinviare alla impostazione di metodo proposta da G. Motzo, La tutela delle situazioni soggettive fra ordinamento comunitario e ordinamento interno, in $\mathrm{Aa} . \mathrm{Vv}$. , I diritti dell'uomo e la partecipazione politica, Torino, 1986.

35 Introduzione allo studio del diritto costituzionale. Le basi del costituzionalismo inglese (1885), 1915, tr.it della versione inglese del 1959, rist. London-New York, 1964, a cura di A. Torre, Bologna, 2003, 162 s che, come ricorda oggi P. Leyland, Introduzione al diritto costituzionale del Regno Unito, Torino, 2005, 7, "propone la spiegazione forse più esauriente delle nozioni-cardine di sovranità parlamentare e di principio della rule of law".

36 A.V. Dicey, op.ult.cit., 165 s., e 171 ss. per i singoli diritti di libertà.

37 Op.ult.cit., $168 \mathrm{s.}$

38 Per semplicità richiamo soltanto, a titolo di esempio, E. Santoro, Rule of law e "libertà degli inglesi". L'interpretazione di Albert Venn Dicey, in P. Costa, D. Zolo (a cura di), Lo Stato di diritto. Storia, teoria, critica, Roma, 2002, 173 ss. U. Mattei, II modello di common law, $2^{a}$ ed. con la collaborazione di L. Antoniolli Deflorian, Torino, 2004, 3 ss.; P. Leyland, op.ult.cit., 27 ss., 139 ss.; A. Torre, Introduzione. Dicey, o della "constitutional morality», in A.V. Dicey, Introduzione, cit., XV ss.; Id., Regno Unito, Bologna, 2005, spec. 32 s., 50 s., 109 ss.; F. Palermo, La forma di Stato, cit., spec. 57 ss. Ma si veda già la dettagliata analisi concreta condotta da W. Wade, H. Ragnemalm, P.L. Strass, Administrative Law. The problem of justice, I, Milano, 1991, 5-234.

39 Riprendo qui l'approccio di metodo proposto nel citato saggio di S.P. Panunzio, I diritti fondamentali e le Corti, cit., al quale rinvio per la ricostruzione critica e le argomentazioni di fondo a sostegno di questa scelta. II saggio contiene inoltre la più dettagliata analisi delle problematiche connesse all'adesione alla CEDU da parte della UE, soprattutto in relazione ai rapporti tra le due Corti.

40 Lo notava già dieci anni fa M. Cartabia, Principi inviolabili e integrazione europea, Milano, 1995, spec. 21 ss., 29 ss., 39 ss., 46 ss. E si veda ora l'approfondita ricostruzione critica proposta da S.P. Panunzio, I diritti fondamentali e le Corti, cit., spec. Parte IV, ricognitiva anche della più recente giurisprudenza a conferma di questa tendenza.

$41 \S 6$ ss. della Parte IV.

42 lbidem, $\S 5$.

${ }^{43}$ Riflettevo su questo problema già nel mio / diritti fondamentali, cit., fin dalla Introduzione.

44 Op.loc.ult.cit., § 3.

45 Sul rischio di veder scavalcata la Corte costituzionale in singoli episodi concreti in cui, sulle stesse vicende, quest'ultima ha negato una protezione adeguata ai diritti fondamentali rispetto agli standard professati dalla giurisprudenza della CEDU si vedano le osservazioni di G. Zagrebelsky, Corti europee e Corti nazionali, cit., con riferimento, ad esempio, al caso del conflitto giurisprudenziale, tuttora attivo, in materia di art. 41-bis dell'ord. pen. - sul quale si vedano anche l'approfondita analisi e le riflessioni critiche di M. Ruotolo, Diritti dei detenuti e Costituzione, Torino, 2002, 218 ss. - o di concreta applicazione dell'art. 68 Cost.it. per il rischio di compromissione del diritto ad un'effettiva tutela giurisdizionale dei diritti. Ma sul punto si v., ancora, infra.

46 Mi limito qui a richiamare la completa analisi contenuta nel bel saggio di C. Focarelli, Equo processo e Convenzione europea dei diritti dell'uomo, Padova, 2001.

47 L. n. $15 / 2005$.

48 Cons. Stato, IV, sent. n. 1559 del 2004. Sono grato a Giacinto della Cananea per avermela segnalata.

$49 \mathrm{Si}$ tratta del comma 2 del citato art. 21-octies, a tenore del quale "Non è annullabile il provvedimento adottato in violazione di norme sul procedimento o sulla forma degli atti qualora, per la natura vincolata del provvedimento, sia palese che il suo contenuto dispositivo non avrebbe potuto essere diverso da quello in concreto adottato. II provvedimento amministrativo non è comunque annullabile per mancata comunicazione dell'avvio del procedimento qualora l'amministrazione dimostri in giudizio che il contenuto del provvedimento non avrebbe potuto essere diverso da quello in concreto adottato". 
50 Per una prima ricognizione della giurisprudenza amministrativa in applicazione delle nuove disposizioni della legge sul procedimento amministrativo si v. M. Betzu, G. Coinu, La riforma della legge n. 241/90, in www.costituzionalismo.it/articolo.asp?id=190.

51 Ritenendo "necessario che il rapporto controverso sia ascrivibile a fattispecie già disciplinate con efficacia diretta in sede comunitaria e che la piena attuazione dei diritti conseguentemente costituiti in capo ai cittadini risulta impedita da una norma interna che ne ostacoli la necessaria e completa protezione giurisdizionale".

52 Si veda, infatti, l'art. II-101 del Trattato costituzionale che declina le regole del "diritto ad una buona amministrazione". Sul punto, diffusamente, D.U. Galetta, I/ diritto ad una buona amministrazione europea come fonte di essenziali garanzie procedimentali nei confronti della pubblica amministrazione, in Riv.it.dir.pubbl.com., 2005, 819 ss.

53 L. cost. 23 novembre 1999, n. 2.

54 Sulle garanzie costituzionali del giusto processo e sulla specifica vicenda qui richiamata rinvio alla completa ed esauriente analisi critica di G. Ferrara, Garanzie processuali dei diritti costituzionali e «giusto processo», in Rass.parl., 1999, 539 ss., spec. 545 ss.

55 Il riferimento va alla sentenza n. 361 del 1998, in Giur.cost., 1998, 3083 ss., con note di M. Scaparone, G. Gemma e R. Pellati, N. Zanon (dal titolo evocativo La Corte, il legislatore ordinario e quello di revisione, ovvero del diritto all'«ultima parola" al cospetto delle decisioni d'incostituzionalità, ivi, 3169 ss.) e P. Ventura, sull'illegittimità costituzionale dell'art. 513 c.p.p. nel testo riformato dal legislatore ordinario proprio al fine di impedire l'utilizzabilità processuale delle prove acquisite in violazione delle regole ora contenute nel testo del nuovo art. 111 Cost. Sul punto ancora, diffusamente, G. Ferrara, op.loc.ult.cit;; nonché D. Bifulco, II "giusto processo" tra revisione della Carta fondamentale e dinamiche della costituzione materiale, in Rass.parl., 2002, 515 ss.

56 Ma si vedano anche gli art. 5 e 6 della Convenzione europea dei diritti dell'Uomo.

57 Ce lo ricorda, ora, F. Palermo, La forma di Stato, cit., spec. 85 ss., 231 ss.

58 Per un'analisi di sistema rinvio al saggio di G. Morbidelli, La tutela giurisdizionale dei diritti nell'ordinamento europeo, in AIC, Annuario 1999. La Costituzione europea, Padova, 2000, 359 ss. Sulle garanzie giurisdizionali dei diritti nel sistema della Carta UE si vedano i saggi di R. Romboli, A. Spadaro, M. Carrillo, M. Cartabia, A. Celotto, M. Revenga Sánchez e G. Silvestri nel volume Aa.Vv., Riflessi della Carta europea dei diritti, cit., 107 ss.

59 Si v. in proposito F. Sorrentino, Le garanzie costituzionali dei diritti. Lezioni raccolte da R. Calvano, Torino, 1998, 20 ss., 136 ss.

60 Così con riferimento alla loro indipendenza, al potere di applicazione diretta del diritto comunitario, alla competenza a sollevare questioni pregiudiziali dinanzi alla Corte di giustizia ed ai requisiti formali necessari per la corretta rimessione di tali questioni, ecc. Sul punto, ora, diffusamente F. Fernández Segado, El juez nacional como juez comunitario europeo. Sus consecuencias constitucionales, in Revista peruana de Derecho público, 2004, 19 ss.

61 E. Santoro, Rule of law, cit., 188.

62 Op.ult.cit., 190.

63 Il riferimento va, naturalmente, al caso Cordova c. Italia, sentt. CEDU, 30 gennaio 2003, in cause 40877/98 e 45649/99, di condanna per denegato accesso all'autorità giudiziaria nel caso di strumentale ed arbitraria applicazione dell'istituto dell'immunità parlamentare.

64 II riferimento va, naturalmente, al caso Cordova c. Italia, sentt. CEDU, 30 gennaio 2003, in cause 40877/98 e 45649/99, di condanna per denegato accesso all'autorità giudiziaria nel caso di strumentale ed arbitraria applicazione dell'istituto dell'immunità parlamentare. Si veda l'ordinanza della Corte costituzionale n. 357 del 2005 in merito alla pretesa immunità dell'ex Presidente della Repubblica Francesco Cossiga dalla giurisdizione civile italiana, in G.U., 1a serie speciale, n. 40 del 5 ottobre 2005, 22 ss.

Recebido para publicação 22/11/2007

Aceito para publicação 05/12/2007 